\title{
Gender differences in the comorbidity of neurological and psychological disorders in a large clinical sample of children
}

\author{
Valerie Brandt, Elisa Napoleone and Praveetha Patalay
}

\section{Summary}

This study aimed to establish rates and gender patterns of 25 comorbidities in 1912 children (72\% male) with a neurological disorder and a comparison group ( $n=40718,45 \%$ male) from a large clinical records data-set in child mental health services in the UK with clinician-recorded data on neurological and psychological conditions. Obsessive-compulsive disorder, oppositional defiant/conduct disorders, autism spectrum disorders and intellectual disabilities (also known in UK health services as learning disabilities) occurred significantly more often in both boys and girls with neurological disorders than in the comparison group. Girls with neurological disorders showed a 'male-typic' comorbidity profile.

\section{Keywords}

Neurological disorder; comorbidity; psychological disorder; multimorbidity; gender

\section{Copyright and usage}

(C) The Author(s), 2021. Published by Cambridge University Press on behalf of the Royal College of Psychiatrists. This is an Open Access article, distributed under the terms of the Creative commons Attribution licence (http://creativecommons.org/ licenses/by/4.0/), which permits unrestricted re-use, distribution, and reproduction in any medium, provided the original work is properly cited.
Neurological disorders and neurodevelopmental disorders have been shown to be associated with some psychological disorders (as defined in chapter 5, ICD-10) in youths. ${ }^{1}$ At least $30 \%$ of adults in primary care have also been diagnosed with a mental health condition ${ }^{2,3}$ and studies point to a link between neurological conditions and some psychological conditions in childhood, such as epilepsy and attention-deficit hyperactivity disorder (ADHD). ${ }^{4}$ Even though it is well-known that many neurological and psychological disorders have a gender bias, for example ADHD, autism spectrum disorder (ASD), conduct disorder, tic disorders and Parkinson disease are more commonly diagnosed in males, whereas anxiety disorders, mood disorders and multiple sclerosis are more commonly diagnosed in females, ${ }^{5}$ little is known about gendered comorbidity patterns between neurological and mental conditions. This study investigates the co-occurrence of a large number of psychological symptoms in children with a neurological disorder in a clinical sample from child mental health services. We also establish gender patterns of psychological comorbidities in children and adolescents with neurological disorders.

\section{Method}

\section{Participants}

The data are from the clinical records of statutory and voluntary child and adolescent mental health services taking part in the Child and Young People's Improving Access to Psychological Therapies (CYP IAPT) service transformation programme in England. The clinical records were collected April 2011-October 2015. Greater detail about these data are reported elsewhere. ${ }^{6}$ In this period, data on $n=122923$ patients were recorded; $n=43156$ included data on the Current View tool, ${ }^{7}$ where clinicians indicate the patients' presenting problems (e.g. ADHD symptoms).

Participants between 3 and 18 years were included in this study (excluding $n=526$ : 366 under 3 years, 116 over 18 years old and 44 with missing age), resulting in an analysis sample of $n=42630$ (46.2\% male), mean age of those with a neurological disorder 11.02 years (s.d. $=3.38$ ); comparison group mean age 12.41 (s.d. $=3.52)$

\section{Measures}

\section{Clinician-reported presenting problems}

Clinicians indicated the child's presenting problems on the Current View tool, ${ }^{7}$ which consists of a list of psychological diagnostic categories (e.g. depression; see the supplementary material for full list available at https://doi.org/10.1192/bjo.2021.53) rated on a severity scale (none, mild, moderate, severe). It also comprises a list of 14 further neurological and developmental disorders such as 'PDD [pervasive developmental disorder]/autism/Asperger's', 'learning disabilities' and 'neurological disorders' (e.g. tics/ Tourette, cerebral palsy, speech and language disorders, e.g. apraxia of speech; common causes: brain injury, tumour) for which clinicians indicate if they are present or not (yes/no).

To ensure the quality of the data, clinicians are provided with detailed guidance on how to complete this tool, which includes definitions and descriptions for each item and descriptors of the response options. ${ }^{7}$ Details of the list of conditions and examples, as provided on the tool, are available in the Supplement material. Based on clinicians' assessments using this tool, 1912 records were identified as being for individuals with a neurological disorder ( $72 \%$ male). The remaining 40718 records (45\% male) constitute the clinical comparison group in this study.

\section{Analysis}

The prevalence of 25 different types of presenting problems in children with a neurological disorder is compared with prevalence rates in the clinical comparison group (overall and by gender, $\chi^{2}$-tests).

\section{Ethics statement}

The data used in this study are patient records and specific consent or ethical permission was not required to conduct this analysis. All data management and confidentiality protocols governing the use of 

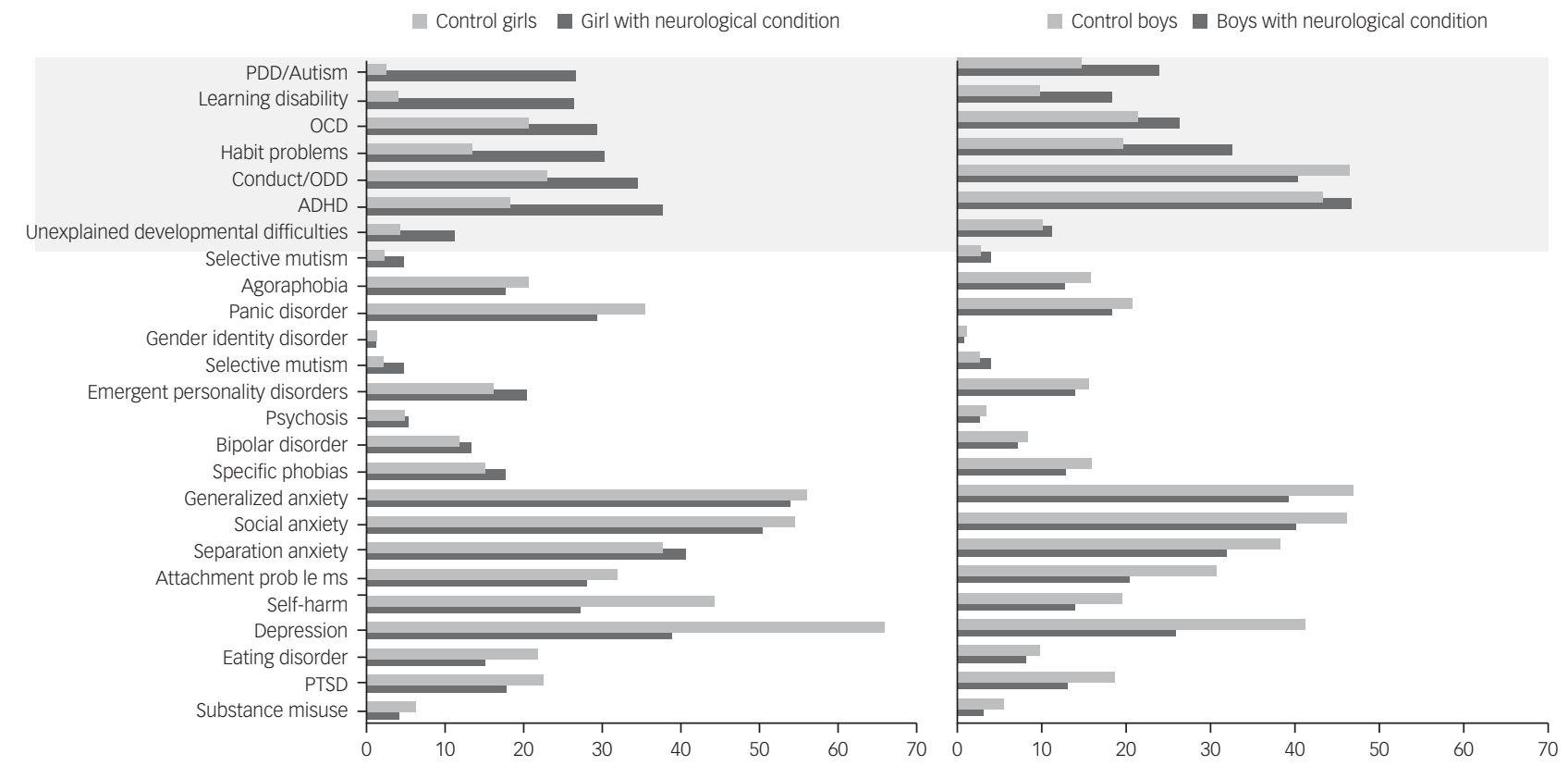

Fig. 1 Rates of psychological disorders in the neurological disorder group and comparison group separately by gender. The findings suggest that the eight symptom domains in the grey shaded area are specifically comorbid with neurological disorders. The remaining disorders are coexisting disorders and occur at increased rates in the clinical comparison sample.

PDD, pervasive developmental disorder; OCD, obsessive-compulsive disorder; ODD, oppositional defiant disorder; ADHD, attention-deficit hyperactivity disorder; PTSD, post-traumatic stress disorder.

the data-set were followed. The manuscript was prepared according to the STROBE guidelines.

\section{Results}

Clinicians reported no other presenting problems in $23 \%$ of individuals with neurological disorders, with $77 \%$ demonstrated symptoms of at least one psychological disorder.

Symptoms of ADHD, conduct disorder/oppositional defiant disorder (ODD), habit problems, obsessive-compulsive disorder (OCD), intellectual disability (also known as learning disability in UK health services), ASD and unexplained developmental disorders were significantly more common in individuals with neurological disorders than the comparison group (Fig. 1, which includes details by gender, and Supplementary Table 1). Symptoms of depression, anxiety, self-harm, substance misuse, attachment problems and eating disorder were less frequent in individuals with neurological disorders compared with the comparison group.

The disorders with the highest co-occurrence with neurological disorders were intellectual disabilities (14.5\%), followed by ASD $(12.8 \%)$.

\section{Discussion}

This is the first study to compare gender patterns in mental health symptoms in a large sample of children with neurological disorders to unrelated children of the same age with at least one psychological presenting problem specified by a trained clinician.

Overall, symptoms of ADHD, OCD/habit problems, ASD and intellectual disability were more common in children with neurological disorders than in the comparison group, whereas depression and anxiety symptoms were less common. The results are partly in line with a previous study, showing an association between neurodevelopmental disorders and ADHD/behaviour problems. ${ }^{1}$ There was a slight difference in mean age between the groups with and without neurological disorder. This could, at least partially explain differences in comorbidities with a later onset, such as depression, self-harm, agoraphobia and substance misuse.

\section{Gender patterns}

A unique contribution of this study is the investigation of gender patterns in neurological-mental disorder comorbidities, which has received little attention to date. Girls with neurological disorders showed a similar comorbidity pattern to boys with neurological disorders (even though the typical gender differences in comorbidities can be found within the neurological disorders group), with girls with neurological disorders displaying a male-typical comorbidity pattern (increased occurrence of ADHD, ODD, ASD, intellectual disability; decreased occurrence of depression, self-harm and eating disorders). This raises the question of whether this set of disorders have similar aetiology.

\section{Strengths and limitations}

The main strengths of this study are the use of a large, detailed clinical data-set of patient records, permitting much more detailed analyses of patterns and outcomes of comorbid symptoms of neurological disorders in children than has been previously possible.

The use of clinician-reported disorders rather than formal diagnosis is a limitation of the study. Furthermore, the scale in the IAPT only makes it possible to check whether any neurological disorder was present or absent, without further details of what type of neurological disorder, barring further detailed analysis of these conditions. Given the scale of the data-set and it being collected as part of routine clinical care, the Current View tool aimed to record conditions based on expert clinical judgement, without the burden of diagnostic interviews across all conditions. This approach is likely to have led to higher recorded prevalence across all disorders in 
our study. However, the similar levels of comorbidities found in this study as previous investigations in clinical samples ${ }^{8,9}$ provides support for the utility and validity of the approach taken. The setting of the study, mental health services data, precludes conclusions being extended to the co-occurrence of these conditions in the general population. Owing to the relatively low prevalence of some of these conditions, very large population-based studies would be necessary to examine this in the general population. Lastly, given the use of health records we cannot differentiate between gender at birth and self-identified gender as there might be variation in what is recorded by services and we used this variable as available.

\section{Implications}

Psychiatric conditions of a neurodevelopmental nature such as $\mathrm{ADHD}, \mathrm{ASD}$ and intellectual disability are more likely to be comorbid in children with neurological conditions; this is especially stark in females where these conditions are all less likely to occur; highlighting the possibility of shared aetiology between these conditions.

Valerie Brandt $(\mathbb{D}, \mathrm{PhD}$, Center for Innovation in Mental Health, Department of Psychology, University of Southampton, Southampton, UK: Elisa Napoleone, MSc, Child Outcomes Research Consortium (CORC), Anna Freud National Centre for Children and Families, London, UK; Praveetha Patalay (D), PhD, Centre for Longitudinal Studies and MRC Unit for Lifelong Health and Ageing, University College London, London, UK

Correspondence: Praveetha Patalay. Email: p.patalay@ucl.ac.uk

First received 6 Nov 2020, final revision 30 Mar 2021, accepted 13 Apr 2021

\section{Supplementary material}

Supplementary material is available online at https://doi.org/10.1192/bjo.2021.53

\section{Data availability}

Data was collected by the Child Outcomes Research consortium (CORC) and is available at Data was collected by the $\mathrm{C} i \mathrm{c}$
CORC upon written request.

\section{Acknowledgements}

The authors would like to thank all partnerships that took part in the Children and Young People's Improving Access to Psychological Therapies (CYP IAPT) service transformation programme 2011-2015 for providing the data presented here. The authors would also like to thank members of the Child Outcomes Research Consortium (CORC), its committee at the time of writing: Miranda Wolpert, Ashley Wyatt, Mick Atkinson, Kate Martin, Ann York, Alan Ovendon, Duncan Law, Julie Elliot, Isobel Fleming - and the CORC team at the time of writing (including E.N): Julian Edbrooke-Childs, Benjamin Richie, Kate Dalzell, Jenna Jacob, Jenny Bloxham, Victoria Zamperoni, Carin Eisenstein, Meera Patel, Andy Whale, Alison Ford, Sally Marriott, Lee Atkins, Danielle Antha, Rebecca Neale.

\section{Author contributions}

V.B. conceptualised and designed the study, drafted the initial manuscript, and reviewed and revised the manuscript. E.N. prepared the data-set, analyse the data and reviewed and revised methods and reviewed and revised the manuscript. All authors approved the final manuscript.

\section{Funding}

P.P. is supported by a Wellcome Trust Institutional Strategic Support Fund (ISSF3/ H17RCO/ NG1), V.B. is supported by the AMS with a springboard award.

\section{Declaration of interest}

None.

\section{References}

1 Merikangas KR, Calkins ME, Burstein M, He JP, Chiavacci R, Lateef T, et al. Comorbidity of physical and mental disorders in the neurodevelopmental genomics cohort study. Pediatrics 2015; 135: e927-38.

2 Druss BG, Walker ER. Mental disorders and medical comorbidity. Synth Proj Res Synth Rep 2011; 21: 1-26.

3 Roca M, Gili M, Garcia-Garcia M, Salva J, Vives M, Garcia Campayo J, et al. Prevalence and comorbidity of common mental disorders in primary care. J Affect Disord 2009; 119: 52-8.

4 Rodenburg $\mathrm{R}$, Stams GJ, Meijer AM, Aldenkamp AP, Dekovic $M$ Psychopathology in children with epilepsy: a meta-analysis. J Pediatr Psychol 2005; 30: 453-68.

5 Thibaut $F$. The role of sex and gender in neuropsychiatric disorders. Dialogues Clin Neurosci 2016; 18: 351-2.

6 Wolpert M, Jacob J, Napoleone E, Whale A, Calderon A, Edbrooke-Childs J. Child- and Parent-reported Outcomes and Experience from Child and Young People's Mental Health Services 2011-2015. CAMHS Press, 2016.

7 Jones M, Hopkins K, Kyrke-Smith R, Davies R, Vostanis P, Wolpert M. Current View Tool Completion Guide. CAMHS Press, 2013.

8 Hirschtritt ME, Lee PC, Pauls DL, Dion Y, Grados MA, Illmann C, et al. Lifetime prevalence, age of risk, and genetic relationships of comorbid psychiatric disorders in Tourette syndrome. JAMA Psychiatry 2015; 72: 325-33.

9 Huisman-van Dijk HM, Schoot R, Rijkeboer MM, Mathews CA, Cath DC. The relationship between tics, OC, ADHD and autism symptoms: a crossdisorder symptom analysis in Gilles de la Tourette syndrome patients and family-members. Psychiatry Res 2016; 237: 138-46.

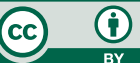

